\title{
Non-invasive Device to Lessen Tremors in the Hands due to Parkinson's Disease
}

\author{
Juan Hinostroza-Quiñones ${ }^{1}$ \\ Facultad de Ciencias e Ingeniería \\ Universidad de Ciencias y Humanidades \\ Lima, Peru
}

\author{
Manuel Vasquez-Cunia ${ }^{2}$ \\ Facultad de Ciencias e Ingeniería \\ Universidad de Ciencias y Humanidades \\ Lima, Peru
}

\begin{abstract}
One of the severe neurological disorders that affects the central nervous system is Parkinson's disease, which causes that patients can not perform routine tasks such as eating and writing. According to statistical data, there are more than $\mathbf{1 0}$ million people in the world who suffer from this disease and the Latin American nation of Peru is no stranger to this, since approximately 30 thousand people suffer from it. Until today there is not a cure for this disease; however, there are different chemical, biological and electronic methods that help to improve the quality of life of patients with this disease. This research aims to design a low-cost device that is able to diminish tremors in patients with Parkinson. The non-invasive device presented and developed in this study will work with the help of 5 vibratory motors and a microcontroller. The vibrations generated by the motors in the patient's wrist will distract the brain and as result the tremors of the hand due to Parkinson's disease will be reduced.
\end{abstract}

Keywords-Parkinson; non-invasive device; vibrations; Arduino

\section{INTRODUCTION}

In recent years, one of the silent diseases that has been increasing, and that most of the people do not know about, is Parkinson's disease (PD), which is the second most frequent neurodegenerative condition after Alzheimer. Parkinson's disease is a neurodegenerative disorder that affects approximately more than 10 million people around the world [1]. These cases particularly affect people aged 60 and over, with certain cases involving young people and adults. In Peru, based on the statistical data of the National Institute of Neurological Sciences (INCN), it has been indicated that 30,000 people suffer from this disease and each year approximately 3,000 cases are reported [2].

Parkinson's symptoms include increased quivering movements, tremors, muscle stiffness, slow movement, and motion irregularity. All of these symptoms cause the patient to harm their daily routines, for instance, in holding certain objects that are necessary to lead a comfortable quality of life.

It is well known that each of the motor functions of the human body is produced thanks to the electrical stimuli that the brain emits, and these stimuli are transmitted by nerve cells (neurons) [3]. Many assistive devices are now available for people with PD; most of them are portable and effective in suppressing tremors. Blended portable robots and portable orthopedic exoskeletons are prominent in the recovery of patients with PD [4], [5], [6], [7]. An Imperial University of London research team has designed an exoskeleton that uses DC motors and a mechanical arrangement of springs to keep the hand steady [8]. The orthosis of the semi-active arm tremor is one of the new developments in the area of the tremor suppression [9], [10]. This device uses an intelligent fluid and its properties can be varied according to the intensity of the tremor, thus damping the hand tremor. Listenme is a device that helps PD patients who present difficulty in walking. Through small auditory signals that reach the brain and the subconsciousness, this device returns the patient the capacity to rotate the body without falling, proving that it eliminates the frozen gait [11]. On the other hand, gyro gloves are devices that suppress tremors by using the principle of a gyroscope, that is, a set of rotating discs which serves to counteract the intensity of the hand tremor [12], [13]. The vast majority of portable cataclysmic suppression devices are costly or, at worst, unavailable to patients with PD living in developing countries such as Peru, where technology lags far behind developed countries.

The present research proposes the design of a low-cost device to reduce wrist tremors of PD patients. This portable device will be able to generate vibrations by means of small vibratory motors. In this way, it will be possible to achieve comfort and calm in patients suffering from this disease so that they can carry out their daily routines without the need of others' external help.

\section{IMPLEMENTATION OF THE PROTOTYPE}

To obtain the expected results, and to reduce the tremors in the hands, the vibratory motors need to be placed near the ulnar and the median nerves, since they are the ones that will control the hand and forearm in greater proportion. Once the device fulfills its function of reducing the tremors, it will automatically shut down as there will be a vibratory sensor next to it that manages the vibration range, so that the patients feel in optimal conditions to perform their daily routines.

\section{A. Design}

Once the biological parameters to be analyzed are identified, we carried out the design of the system; the diagram of this is shown in Fig. 1. The component used for this device are relatively inexpensive and can be obtainable easily in the market.

These are the following components to be used:

- Vibratory motors (5 units) 


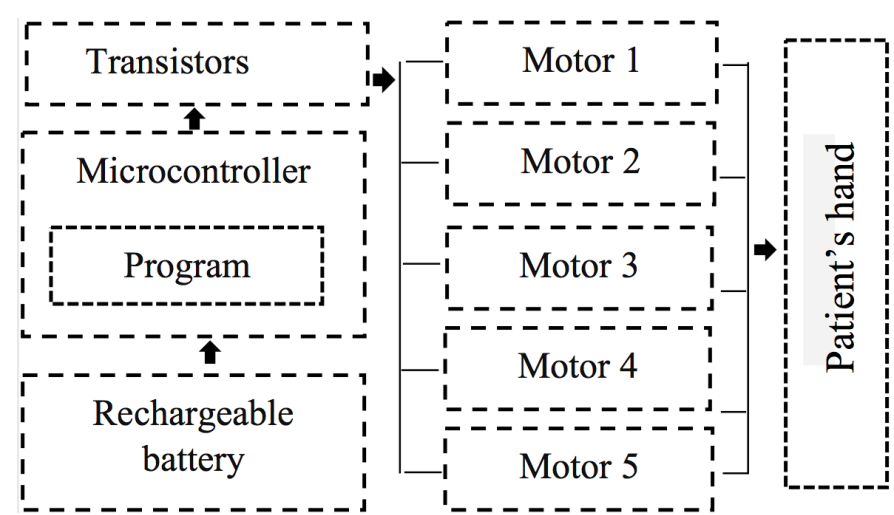

Fig. 1. Diagram of the Device to Lessen Tremors due to PD.

- Two-pole transistor

- Microcontroller

- $\quad$ Rechargeable Battery (6-9 V)

All these components are integrated by an Arduino Nano ATmega 328p microcontroller, which will process the program previously uploaded, and will send signals to the motors to cause the vibrations needed. Since five vibration motors are being used, and there is a risk that the microcontroller will be damaged by the increase of the current supplied to these, a 2 N2222 transistor is put into use.

\section{B. Electronic Components}

Arduino Nano: A small card with a microcontroller used to store the developed algorithm to control the system.

2N2222 Transistor (Fig. 2): This component is used to account for the current needed by the vibratory motors.

Vibration mini motor (Fig. 3): It is a DC motor that receives electrical pulses to convert them into vibrations. A total of 5 are used.

The development of the program was mainly based on three random vibration ranges: if the first value is equal to the second and the third ones as well, the five motors are activated; otherwise, we need to perform a second evaluation. If the second random value is equal to the third one, the five motors must be deactivated, otherwise, one last block of code is executed: the engines of equal values of random variables 1 and 2 must be activated. To understand this algorithm better, the flow chart is shown in Fig. 4. The algorithm consists of random ranges that are defined through the $\mathrm{X}, \mathrm{Y}$ and $\mathrm{Z}$ variables.
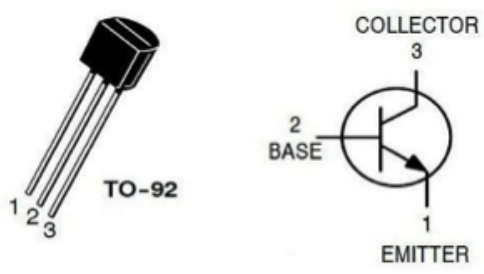

Fig. 2. 2N2222 Transistor.

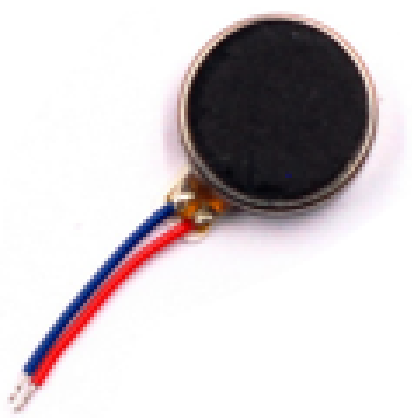

Fig. 3. DC Vibration Mini Motor

\section{Simulation of the Prototype}

In Fig. 5, the simulation of the prototype done in the software Proteus is shown. This simulation is accompanied by an Arduino Nano, which function to send signals to each vibratory motor. For this purpose, the program developed was compiled at the beginning before conducting the corresponding tests. Additionally, it can be noted that in the simulation each of the vibratory motors are connected to the transistor 2 N2222. The transistor base is connected to one of the ports assigned in the program so that the Arduino can recognize it to send the

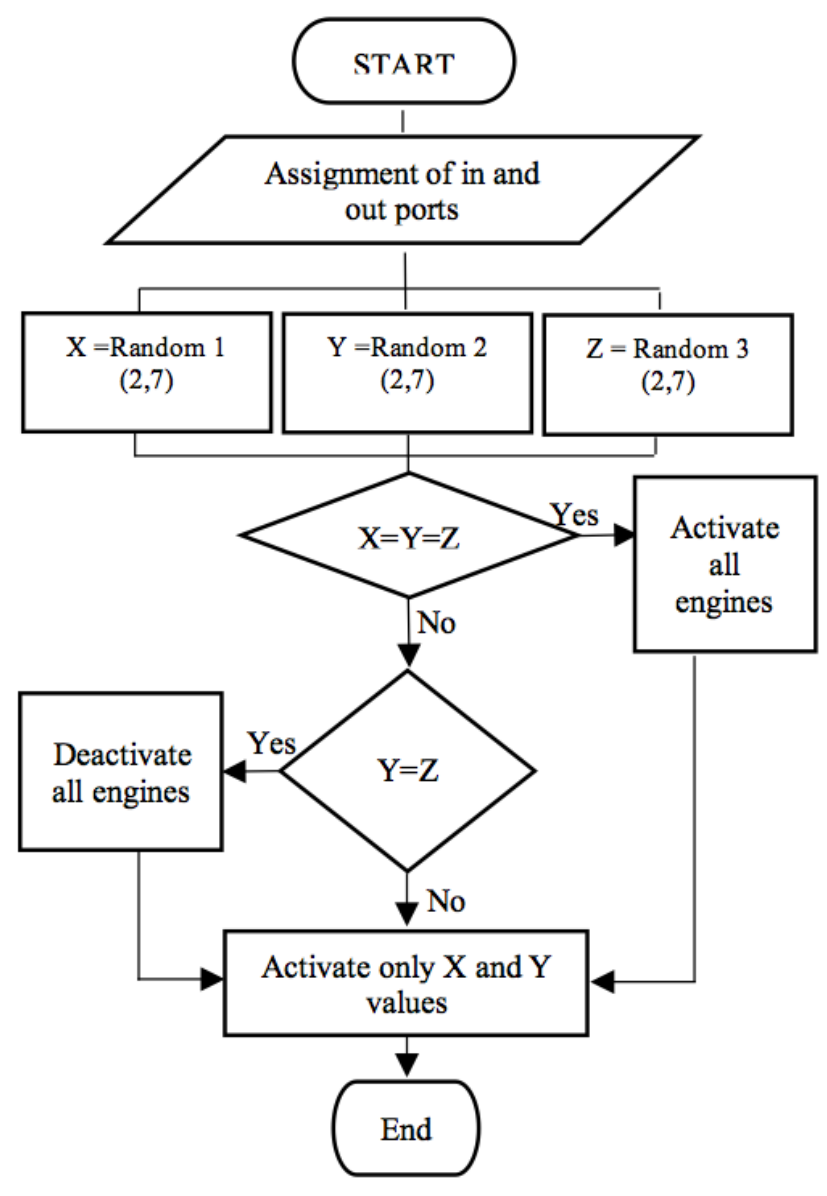

Fig. 4. Flowchart of the Program. 


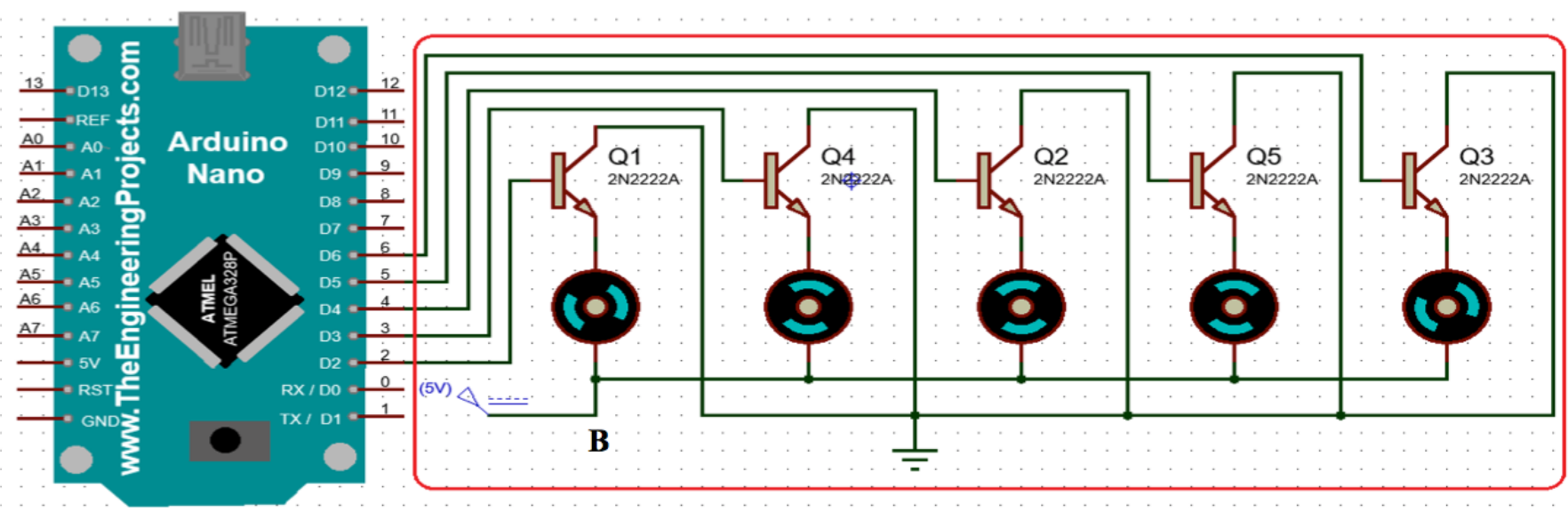

$\mathbf{A}$

Fig. 5. Simulation of the Device in Proteus.

signals for the motors to work. Finally, in the simulation can be seen the vibratory motors that are in charge of receiving the signals sent from the Arduino to perform the necessary vibrations.

\section{RESULTS AND DISCUSSION}

Research shows that Parkinson's disease affects adults aged 35 years and older and it has not a definitive cure until today, reason why the proposed low-cost device in this work.

Fig. 6 shows the implemented prototype, with all components and a battery to have it functioning. The vibration motors are attached to a small elastic band, which is of $5 \mathrm{~cm}$ wide and has a length of $30 \mathrm{~cm}$. The device is put on the wrist and attached with the help of a strap secured with velcro.

The arm nerves can feel the vibrations that are generated by the motors, which is why the brain focuses more on random vibration patterns and ignores the hand tremors generated by the Parkinson's disease. The arms nerves are the receptors that work with the brain to regulate voluntary movements. The

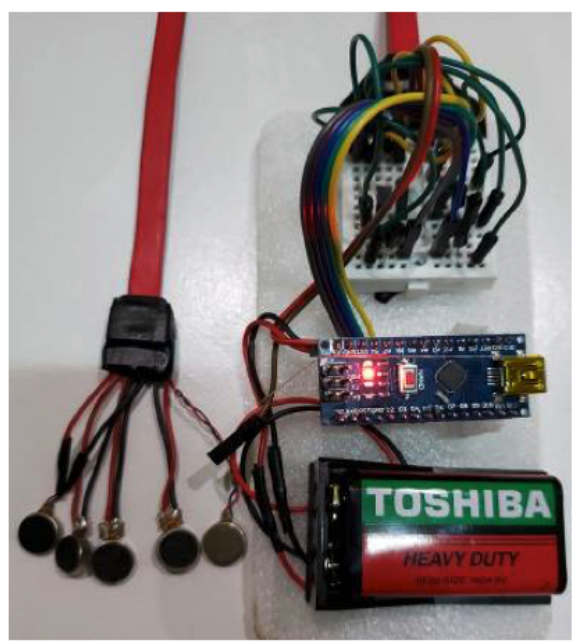

Fig. 6. Implemented Prototype.

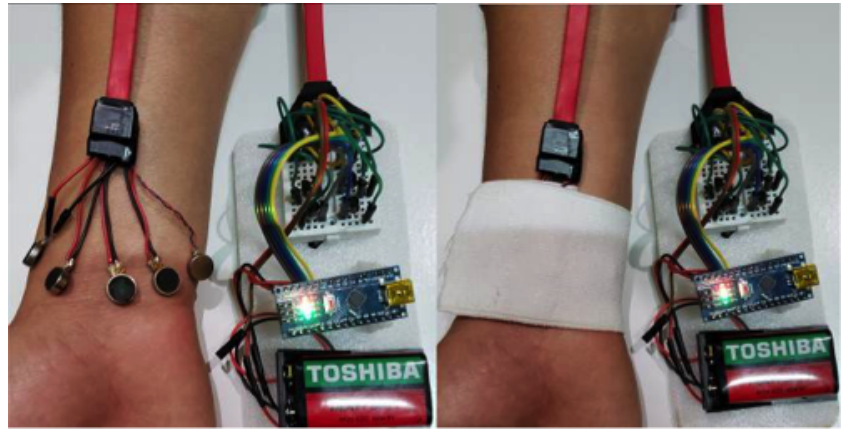

Fig. 7. The Prototype in Test Mode.

effect of the vibrations will depend on the seriousness of the tremors. The choice of vibration pattern will depend from one patient to another. A random vibration may be more effective than the autonomous vibration produced by the motors.

Fig. 7 shows the tests of the device performed on a person. A random and rhythmic vibration pattern has been generated and applied to the wrist area.

The device was tested on patients with arm tremors, for which there were evaluations from time to time, as Table I shows. Each test was performed for a total period of 20 minutes for each patient, and every 5 minutes they were asked about their sensations. The sensation were classified as: A) no reduction of tremors, B) minimal reduction and C) significant reduction.

On completion of the tests, positive results were obtained by the device. For patients 1 and 3 after the 20 minutes of evaluation there was a significant reduction in the tremors

TABLE I. RESULTS OF THE TEST OF THE PROTOTYPE.

\begin{tabular}{ccccc} 
& $\mathbf{5}$ $\mathbf{~ i n}$ & $\mathbf{1 0} \mathbf{~ m i n}$ & $\mathbf{1 5} \mathbf{~ m i n}$ & $\mathbf{2 0} \mathbf{~ m i n}$ \\
\hline \hline Patient 1 & $\mathrm{~A}$ & $\mathrm{~B}$ & $\mathrm{C}$ & $\mathrm{C}$ \\
Patient 2 & $\mathrm{A}$ & $\mathrm{A}$ & $\mathrm{B}$ & $\mathrm{B}$ \\
Patient 3 & $\mathrm{A}$ & $\mathrm{A}$ & $\mathrm{B}$ & $\mathrm{C}$
\end{tabular}


sensations. In the case of patient 2 the reduction was minimal; thus, not considering this as a positive result. One reason for the aforementioned can be that not all patients react in the same manner to a similar intensity of vibrations. Overall, the initial prototype test showed satisfying results, and test with a big number of patients with Parkinson's disease would be performed in the coming months to improve this device.

To obtain a high effectiveness from the device at the moment of being exposed to tests in a patient with Parkinson's disease, it is proposed to raise the vibration intensity of the motors. However, when the vibrations are raised, it will generate a greater energy consumption, thus it is suggested the use of lithium battery that presents the technical characteristic of lasting longer and of being rechargeable when needed.

The proposed device is intended to be portable, enduring and affordable for the patient with limited financial resources. On the other hand to further lower costs, it is recommended the use of PICs (Programmable Interface Controllers), as these are cheaper than Arduinos and also would lower the dimensions of the device. Additionally, the device presented in this work can be complemented with the use of a smartphone, devices which are nowadays ubiquitous in Peru [14], in order to monitor better the patients.

\section{CONCLUSIONS}

The device presented in this work uses light and portable components so that it can be easily handled to abate tremors from the patient with Parkinson's disease. Moreover, the components used are of very low cost allowing the accessibility to many patients with scarce resources, as happens in many developed nations like Peru.

The tests in three patients have shown positives results, however, follow-up studies will be undertanken with a much bigger sample. Also, the identification of the main nerves of the arm that are interconnected with the brain will allow us to determine accurately the position of where the vibratory motors will be attached to the patient's wrist.

\section{REFERENCES}

[1] N. Ball, W.P. Teo, S. Chandra, and J.Chapman, "Parkinson's Disease and the Environment," Frontiers in Neurology, vol. 10, 2019.

[2] El Peruano, Parkinson afecta a 30,000 peruanos. [Online]. Available: https://www.elperuano.pe/noticia-parkinson-afecta-a-30000-peruanos77467.aspx

[3] BBC, Nervous system - Nerve Cells and Nerves. [Online]. Available: https://www.bbc.co.uk/science/humanbody/body/factfiles/nervecellsandnerves/nerve_cells_and_nerves.shtml

[4] D. Wright, K. Nakamura, T. Maeda, K. Kutsuzawa, K. Miyawaki and K. Nagata, "Research and development of a portable device to quantify muscle tone in patients with Parkinsons disease," 2008 30th Annual International Conference of the IEEE Engineering in Medicine and Biology Society, Vancouver, BC, 2008, pp. 2825-2827.

[5] R.T. Meyer, and Y. Sergeeva, "Mixed-reality assistive robotic power chair simulator for Parkinson's tremor testing," Medical Engineering \& Physics, 2020.

[6] Y. Miyake, "Interpersonal Synchronization of Body Motion and the Walk-Mate Walking Support Robot," in IEEE Transactions on Robotics, vol. 25, no. 3, pp. 638-644, June 2009.

[7] R.W. Horst, "A bio-robotic leg orthosis for rehabilitation and mobility enhancement," 2009 Annual International Conference of the IEEE Engineering in Medicine and Biology Society, Minneapolis, MN, 2009, pp. 5030-5033.

[8] A. Burton, "Expecting exoskeletons for more than spinal cord injury," The Lancet Neurology, vol. 17, pp. 302-303, 2018.

[9] S. Costa, J. Bourget, G. Jablonski, L. Maire, A. Rabelo, M.I. Okereke, L. Chagas, A. Pereira, and A. Andrade, "Ergonomic Evaluation of an Active Wrist Orthosis for the Treatment of Muscular Rigidity in Individuals with Parkinson's Disease," in XXVI Brazilian Congress on Biomedical Engineering, vol. 70, 2019.

[10] S.M. Hashemi, M.F. Golnaraghi, and A.E. Patla,"Tuned vibration absorber for suppression of rest tremor in Parkinson's disease," Medical and Biological Engineering and Computing, vol. 42, pp. 61-70, 2004.

[11] El Tiempo, Colombianos crean gafas para el Parkinson. [Online]. Available: https://www.eltiempo.com/archivo/documento/CMS-14582977

[12] H. Dai and L. T. D'Angelo, "Quantitative assessment of tremor during deep brain stimulation using a wearable glove system," 2013 IEEE International Workshop of Internet-of-Things Networking and Control (IoT-NC), New Orleans, LA, 2013, pp. 53-57.

[13] E. Rovini, C. Maremmani and F. Cavallo, "How Wearable Sensors Can Support Parkinson's Disease Diagnosis and Treatment: A Systematic Review," Frontiers in Neuroscience, vol. 11, 2017.

[14] C. Sotomayor-Beltran and L. Andrade-Arenas, "A spatial assessment on internet access in Peru between 2007 and 2016 and its implications in education and innovation," 2019 IEEE 1st Sustainable Cities Latin America Conference (SCLA), Arequipa, Peru, 2019, pp. 1-4. 